\title{
Real-Time Binding Kinetics Monitored with Surface Plasmon Resonance Imaging in a Diffusion-Free Environment
}

\author{
Roberta D'Agata ${ }^{1}$, Giuseppe Grasso ${ }^{1}$ and Giuseppe Spoto ${ }^{*}, 1,2$ \\ ${ }^{I}$ Dipartimento di Scienze Chimiche, Università di Catania, Viale Andrea Doria 6, 95125, Catania, Italy \\ ${ }^{2}$ Istituto Biostrutture e Bioimmagini, CNR, Viale A. Doria 6, Catania, Italy
}

\begin{abstract}
Surface Plasmon Resonance Imaging (SPRI) is an optical technique emerged as a powerful tool for the simultaneous monitoring of interactions of biomolecules arrayed onto gold substrates. To take fully advantage of the SPRI approach a precise control of the fluidic of the analyte solution is imposed. The diffusion between the flowing buffer and the analyte solution which is established within the fluidic system creates a liquid volume where a gradient in the analyte concentration is present. Such gradient is shown to affect the kinetics parameters obtained from the SPRI response. We present results obtained from a new delivery approach based on the use of air bubbles. The advantages offered by the new approach are demonstrated by using two different interacting biomolecular systems: the streptavidin-biotin system and the Ricinus Communis Agglutinin lectin-asialofetuin.
\end{abstract}

\section{INTRODUCTION}

Attention towards the control and the manipulation of fluids in the microfluidic regime started to appear in the literature more than twenty years ago [1], but it is only in the last few years that the number of papers regarding the use of microfluidic networks for manipulating and analyzing biological liquid samples has been rapidly increased $[2,3]$. The main reason behind such rapid development is that microfluidic devices offer potential advantages over conventional biosensor instrumentation. A microfluidic device has one or more channels with at least one dimension less than $1 \mathrm{~mm}$. The reduced dimensions make phenomena such as diffusion, surface tension and viscosity ever more important [3]. This working environment is suitable for lots of different applications such as cell patterning [4] and separations [5], DNA analysis [6], flow injection reaction analysis [7], enzyme reaction kinetics [8] and many others [9]. In recent years, the coupling of microfluidic devices, often made in poly(dimethylsiloxane) (PDMS), with optical techniques such as surface plasmon resonance imaging (SPRI) [10] has shown to be a powerful tool for the simultaneous monitoring of interactions of arrayed biomolecules. SPRI expands the labelfree capability of the standard SPR technique to rapidly evaluate the interaction between an analyte and its bio-specific ligand immobilized on a metal sensor surface $[11,12]$ by allowing a multiplexed approach to the evaluation of biomolecular interactions $[13,14]$. To take fully advantage of the SPRI approach a precise control of both the patterning of biomolecules onto the sensor surface as well as the fluidic of the analyte solution is imposed. In this perspective, the use of microfluidic devices provides an SPRI compatible convenient mean for both manipulating very small amounts of sample as well as for controlling the patterning of a variety of different biomolecules. Microscale conditions induce the

\footnotetext{
*Address correspondence to this author at the Dipartimento di Scienze Chimiche, Università di Catania, Viale Andrea Doria 6, 95125, Catania, Italy; E-mail: gspoto@dipchi.unict.it
}

laminar flow of fluids [3] (the flow of fluids through microchannels is characterized by low Reynolds numbers, usually lower than 100) and optimize the way in which liquids are put in contact to the SPRI sensor surface. Moreover, mixing of fluids in microchannels is diffusion rather than convection controlled [3].

A typical problem found in SPR experiments raises when the speed of the analyte transport from the bulk solution to the ligand immobilized onto the sensor surface is lower than the ligand-analyte interaction [15]. Increased flow rates are often used in order to minimize analyte mass transport effects [16] that otherwise could cause alterations in the kinetics parameters obtained from SPR experiments. Unfortunately, larger amount of sample volumes are required as a consequence of the need to increase the flow rate and to maintain proper contact times between the analyte solution and the SPR sensor surface immobilized biomolecules. For many years there has been a keen interest in finding methods for eliminating the problem of mass-transport [17]. Today, we know that the mass-transport effect is related to the diffusion coefficient of the analyte $(D)$, the flow rate of the bulk solution $(f)$ and the flow cell dimensions [17]. Alternative SPR experimental setups have been also proposed to overcome the above mentioned limitation [18]. For example, miniaturized flow cells, such as those obtained by using microfluidic devices, help in overcoming mass-transport rate limitation thanks to the reduced sample volume involved. Nevertheless, when fast interactions and large molecules are going to be concerned the above methods cannot completely eliminate diffusion processes occurring in the Nernst diffusion layer [19], as well as at the interfaces between fluids which flow through the tubing system.

In this context, some experimental issues such as the way by which the analyte solution is put in contact with the surface become ever more important. In a standard SPR apparatus, the switching process between different solutions that have to enter the SPR flow cell is usually carried out by using computer-controlled actuators and different sample 
loops. Unfortunately this method limits the maximal sample volume to the size of the injection loops, forbidding the possibility to study interactions for long times [20].

In order to solve the above mentioned problems peristaltic pumps are often used in common SPRI experimental setups for flowing solutions into PDMS made microfluidic devices that are in contact with the SPRI metallic sensor surface [13]. The resulting diffusion between the running buffer and the analyte solution which is established within the pump tubing system creates a liquid volume where a gradient in the analyte concentration is present. Such gradient depends not only on $D$ and $f$, but also on the length of the tubing systems and affects the SPRI response in kinetic experiments. The use of air bubbles (ABs) in SPR [18], as well as in SPR coupled with MS [21], has been often applied as a way to separate different solutions. The introduction of an $A B$ between the running buffer and the solution of the analyte in the same buffer has been also proposed as a method to minimize the Nernst diffusion layer in a TIRF rheometer [19].

In the present paper, we focus only on the diffusion processes occurring at the liquid-liquid interface. To work under conditions that are not limited by mass transport, the flow rate was increased to $100 \mu 1 / \mathrm{min}$.

We firstly demonstrate that the diffusion between different solutions occurring inside the tubing system causes a concentration gradient that is detectable by the SPRI system as a characteristic as well as misleading response. Consequently, we introduce the use of ABs in SPRI measurements as a method for obtaining data that are not affected by the above mentioned process. The proposed method is shown to be particularly advantageous in SPRI studies for monitoring real time interactions of molecules with high $D$ values and/or high affinity towards the surface immobilized partner. Considerations about how this phenomenon can affect the quantitative measurements of important parameters such as the kinetic rate constants in the case of different interacting biomolecules are discussed. The streptavidin-biotin interaction, as well as the binding of the Ricinus Communis Agglutinin $\left(\mathrm{RCA}_{120}\right)$ lectin with asialofetuin (ASF) are used as examples to demonstrate the applicability of the new approach to the study of biological molecules. The latter are shown not to be damaged by the ABs transit in the flow cell.

\section{MATERIALS AND METHODS}

\section{Materials}

Reagents were obtained from commercial suppliers and used without further purification. Cystamine dihydrochloride, D-biotin, (+)-biotin N-hydroxysuccinimide ester, Dithiobis succinimidylpropionate (DTSP, Lomant's Reagent), triethanolamine (TEA), Ricinus Communis agglutinin $\left(\mathrm{RCA}_{120}\right)$ and asialofetuin (ASF) were purchased from Sigma-Aldrich; N-hydroxysuccinimidyl ester of methoxypolyethyleneglycol propionic acid (mPEG-NHS, MW=5000) and methoxy-polyethyleneglycol amine (mPEG-NH $\mathrm{N}_{2}$, MW=5000) were purchased from Nektar Therapeutics (USA); wild-type streptavidin (SA) was obtained from Invitrogen (Italy). Ultra-pure water (Milli-Q Element, Millipore) was used for all the experiments. Phosphate buffered saline (PBS) solutions at $\mathrm{pH}=7.4,(\mathrm{NaCl} 137 \mathrm{mM}, \mathrm{KCl} 2.7 \mathrm{mM}$, phosphate buffered $10 \mathrm{mM}$, Amresco) were used in the SPRI experiments. Absolute ethanol (Sigma-Aldrich) and ultrapure water were used for rinsing and for making solutions.

\section{Surface Plasmon Resonance Imaging Apparatus}

All the SPRI experiments were carried out by using an SPR imager apparatus (GWC Technologies, USA) elsewhere described in detail [22].

SPR images were analyzed by using the $\mathrm{V}++$ software (version 4.0, Digital Optics Limited, New Zealand) and the software package Image $\mathrm{J} 1.32 \mathrm{j}$ (National Institutes of Health, USA). SPRI provides data as pixel intensity units on a $0-255$ scale. As specified by the manufacturer, data are converted in percentage of reflectivity $(\% \mathrm{R})$, or $\Delta \% \mathrm{R}$ in the case of difference images, by using the formula:

$\% \mathrm{R}=100 *(0.85 \mathrm{Ip} / \mathrm{Is})$

where Ip and Is refer to the reflected light intensity detected using p- and s-polarized light, respectively. Kinetic experiments were carried out by sequentially acquiring 15 frames averaged SPR images with no time delay between them. A $15 \mathrm{sec}$ delay was used for prolonged kinetic experiments (from 2 to 8 hours). Kinetics data were obtained by plotting the difference in percent reflectivity $(\Delta \% \mathrm{R})$ from selected regions of interest (ROIs) of the SPR images as a function of time. To correct raw SPRI data for nonspecific adsorption and changes in bulk refractive index, $\Delta \% \mathrm{R}$ for each ROI was normalized to the average of the $\Delta \% \mathrm{R}$ measured for the background ROIs obtained from adjacent regions where no specific interactions were expected to occur.

\section{PDMS Microfluidic Channels Fabrication}

Microfluidic channels were fabricated in poly(dimethylsiloxane) (PDMS) polymer as described elsewhere [23]. Briefly, PDMS microchannels having a volume of about 0.7 $\mu 1$ were created by replication from two masters in polyvinyl chloride (PVC), with a pattern of two $(80 \mu \mathrm{m}$ depth, $1.4 \mathrm{~cm}$ length, $600 \mu \mathrm{m}$ width) and four $(80 \mu \mathrm{m}$ depth, $1.4 \mathrm{~cm}$ length, $400 \mu \mathrm{m}$ width) parallel microchannels, featuring circular reservoirs (diameter $400 \mu \mathrm{m}$ ) at both ends of each channel. PEEK tubes (Upchurch Scientific) were inserted in such reservoirs in order to connect the PDMS microfluidic cell to a Masterflex L/S (Cole-Parmer, USA) peristaltic pump, operating at $100 \mu 1 / \mathrm{min}$. Replicas were formed from a 1:10 mixture of PDMS curing agent and prepolymer (Sylgard 184, Dow Corning, USA). The mixture was degassed under vacuum and then poured onto the master in order to create a layer with a thickness of about 3-4 mm. The PDMS was then cured for at least 2 hours at $60{ }^{\circ} \mathrm{C}$ before it was removed from the masters.

\section{SPRI Experiments}

\section{Water-Ethanol (1\%)}

The mixing caused by diffusion processes between different solutions flowing in a tubing system is easily visualized with an SPRI apparatus by performing an experiment where two solutions having different refraction indexes are forced to flow one after the other into the SPRI flow cell. In order to deliver this simple elution sequence, a switching mechanism that allows the change in the eluted solutions has to be coupled to the tubing system and a common way to 
operate when a peristaltic pump is used is to stop the pumping power during the switching step (stopping flow), in order to avoid introduction of $\mathrm{ABs}$ into the tubing system. In Fig. (1) $\Delta \% \mathrm{R}$ is reported as a function of time for a SPRI experiment where a switching between water and a water solution containing ethanol (1\%) was carried out according to the stopping flow method (black line). In the same figure the $\Delta \% \mathrm{R}$ variation over time obtained for the same elution sequence with the introduction of an $\mathrm{AB}$ between each change of fluid is also reported (grey line).

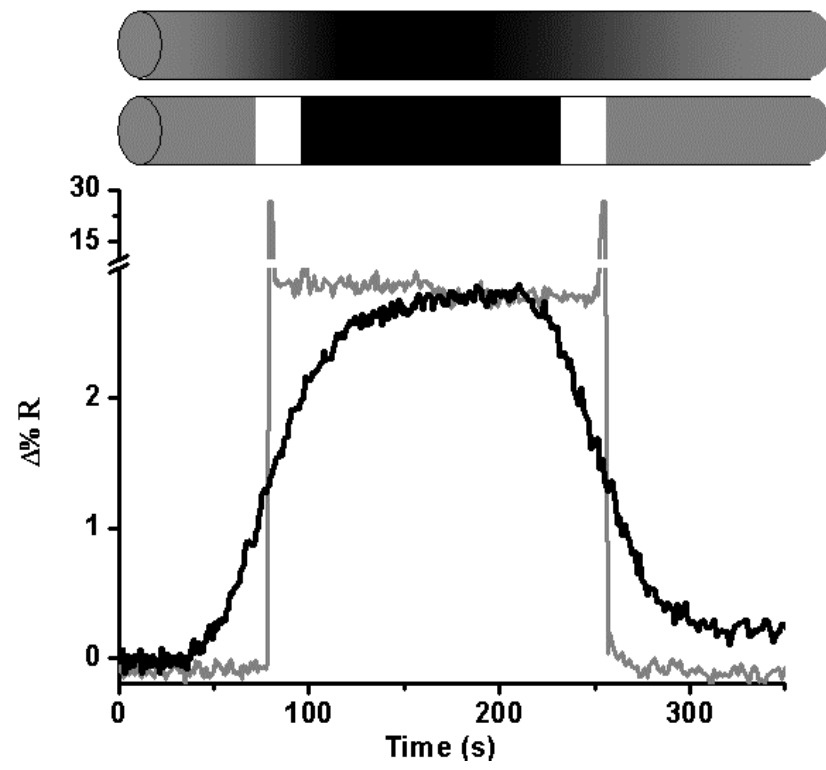

Fig. (1). Percent reflectivity variation over time obtained by switching between water and a water solution containing ethanol $(1 \%)$ with (gray line) and without (black line) the introduction of an $A B$ between each change of fluid (pumping power on for 5 seconds at $100 \mu \mathrm{l} / \mathrm{min}$ in air). The gradient in concentration between the two different solvents $($ water $=$ gray, ethanol $=$ black, $\mathrm{ABs}=$ white) inside the tubing system as it is revealed by the SPRI response is depicted above the two graphs. Note the absence of diffusion processes when $\mathrm{ABs}$ take the place of solvent interdiffusion.

From the SPRI response recorded for this simple experiment it is clear that there is not detectable air diffusion within the two AB separated solutions (Fig. (1), grey line) and therefore the introduction of an $\mathrm{AB}$ during the switching step can in principle allow the acquisition of diffusion-free data.

\section{SA-Biotin}

SA (from Streptomices avidinii) is a homotetrameric protein which binds biotin with high affinity $\left(\mathrm{Ka} \sim 10^{13} \mathrm{M}^{-1}\right)$ [24]. Because of this tight binding, SA has been often used as a capturing molecule for in vitro detection, localization and immobilization of biotinylated biomolecules [25]. The SAbiotin system has been widely applied for a wide range of applications [26], but it also offers bio-analytical advantages as it represents a good model for studying strong biomolecular interactions for which accurate determination of the kinetics binding parameters by SPR is a very challenging task, due to the fast time scale in which such interactions take place [27]. In order to determine the applicability and reliability of the proposed approach, we investigated the interaction of SA with biotin by using an SPRI apparatus coupled with a microfluidic system. The first step of the experiment was the immersion of a bare gold chips (GWC Technologies, USA) in a $0.1 \mathrm{M}$ cystamine water solution (48 hours under inert atmosphere at room temperature) in order to form a self-assembled monolayer on the gold surface. The modified chips were thoroughly rinsed, respectively, with ultra-pure water and ethanol and then dried under a stream of nitrogen. A PDMS polymer mold carrying two parallel microchannels was then physically attached to the cystamine-modified gold surface. A surface pattern was created by flowing the $(+)$ biotin N-hydroxysuccinimide ester (biotin-NHS) solution in PBS $(3.75 \mathrm{mM})$ through the PDMS microchannels over the gold surface.

After the biotin immobilization [28], the PBS buffer solution was flowed into the microchannels until the SPRI signal was stable. At this point, the PDMS polymer mold was removed from the surface and the gold chip was immersed in an $\mathrm{mPEG}-\mathrm{NHS}$ solution ( $4 \mathrm{mM}$ in $0.1 \mathrm{M}$ TEA, $\mathrm{pH}=8$ ) for 2 hours in order to block the unreacted cystamine groups. This mPEG-coated background prevents nonspecific adsorption of SA during the SPRI experiments. In order to deliver SA molecules to the surface, a second set of four PDMS microchannels was then attached to the surface perpendicular to direction of the first biotin microchannel array [29]. The intersection of the latter with the four microchannels was used for monitoring the interaction with SA at four different concentrations $(0.83 \mu \mathrm{M}, 1.66 \mu \mathrm{M}, 3.33 \mu \mathrm{M}, 5.83 \mu \mathrm{M}$ in PBS $\mathrm{pH}=7.4$, see Fig. (2)), while other adjacent areas were considered for subtracting the background signal due to nonspecific protein adsorption interactions [30].



Fig. (2). Difference SPRI image obtained by flowing SA solutions at four different concentrations $(0.83,1.66,3.33$ and $5.83 \mu \mathrm{M})$ in the four different microchannels that are positioned perpendicular to the two ones where the biotin molecules have been previously anchored. The intersection areas are clearly visible as they become brighter as a consequence of the change in the refraction index of the system, registered by the CCD camera as a change in the luminosity of the reflected light. Dark areas of the microchannels nearby the intersection points have been used for subtracting the background signal due to non specific protein adsorption interactions.

Kinetic data were obtained by plotting the $\Delta \% \mathrm{R}$ as a function of time, between the average signals of the intersec- 
tion areas where the biotin molecules were immobilized and the average signals of adjacent areas for mPEG background (Fig. 3). Further details of similar procedures have been described elsewhere [13].

The interaction model for the SA-biotin interaction takes into account the possibility to bind one or two biotin molecules to an SA molecule (bivalent model) [31,32]. This model is justified by the presence of four binding sites in the SA structure. The $D_{2}$ symmetry of the protein structure separates the four subunits as to arrange two of them on one side and the other two on the opposite side of the molecule [33]. Interaction can thus take place between the surface immobilized biotin molecules and one or two of the binding sites present on the SA structure. This mechanism has been widely adopted for modeling deviations from simple firstorder kinetics commonly found in the desorption of SA from biotinylated SAMs [34]. Therefore, there are two key rate constants for the steps involved in the above mechanism. The first-order rate constant for the competitive cleavage of the bond between SA and one molecule of surface-bound biotin and the slower rate constant for the simultaneous dissociation of both SA-biotin bonds or their sequential dissociation. Since the rate constant from the fastest exponential component has been usually reported as a conservative estimate of the rate constants for dissociation of SA from surface-bound biotin [35], the curves are generally fitted with a single first-order exponential decay. The kinetics constant reported as $\mathrm{k}^{\mathrm{app}}$ off in Table $\mathbf{1}$ was obtained by using this approach.

Moreover, although the dissociation proceeds with a faster rate on a surface rather than in a solution, mainly because of entropic reasons [34], the recourse to mutant SA molecules that have a lower affinity towards biotin and therefore dissociate on a faster time scale is often necessary in order to estimate the SA-biotin kinetics binding constants [36]. Alternatively, another approach used for the study of the SA-biotin interaction considers the steady-state part of the binding SPRI curve, reached when association $\left(\mathrm{k}_{\text {on }}\right)$ and dissociation $\left(\mathrm{k}_{\text {off }}\right)$ kinetic rate constants are equal [37]. Both the two mentioned approaches do not consider the initial adsorption phase of SA with biotin. Normally, when the rapid interaction between these two biomolecules is investigated by SPR, an underestimation of the kinetics parameters occurs due to both intrinsic limitations of the technique response as well as the diffusion processes inside the tubing system. In our case, the latter constrain was minimized by the introduction of $\mathrm{ABs}$ in the elution sequence and the advantage of considering all the different portions of the interaction curves (including the initial adsorption phase) could therefore be exploited. In order to validate our results, data obtained from two different SPRI experiments were considered:

SPRI kinetic data for the adsorption of SA (Fig. 3) were fitted with the function resulting from the numerical integration of the differential rate equations for the bivalent model [35] (ClampXP data analysis software [38]). Four different SA concentrations $(0.83,1.66,3.33$ and $5.83 \mu \mathrm{M})$ provided the average values for $k_{\text {on }}$ and $k_{\text {off }}$ shown in Table 1 . Similar experiments were carried out with and without $\mathrm{ABs}$ to check the reliability of the AB-based method. ii) The desorption of the surface bound SA by competitive dissociation with free biotin in solution (1mM biotin in PBS buffer) was followed for several hours and the kinetic data were fitted (Fig. 6) by using a single first-order exponential decay equation as described elsewhere [34,35].

The kinetics parameters obtained by the different approaches are reported in Table $\mathbf{1}$ where a comparison with kinetics parameters available from the literature for analogous systems is also carried out.

\section{$A S F-R C A_{120}$}

Lectins ability to distinguish between variations of oligosaccharide structures makes them perfectly suitable as decoders for carbohydrate-encoded information involved in many biological processes [39] and have been attributed to disease progression, making analysis of saccharide-lectin binding processes important as a diagnostic tool [40]. The lectin from Ricinus Communis Agglutinin $\left(\mathrm{RCA}_{120}\right)$ is a potent cytotoxic protein contained in the seeds of the castor bean Ricinus Communis and it has been shown that $\mathrm{RCA}_{120}$ recognizes $\beta$-linked galactosyl residues of ASF with high affinity $\mathrm{K}_{\mathrm{A}} \sim 1.6 \times 10^{8} \mathrm{M}^{-1}$ [41].

ASF carrying desialylated fetuin side chains, possesses three Gal $\beta_{1,3}$ GalNAc residues for each polypeptide chain. Two of them are close to each other while the third is remotely positioned [42].

SPRI experiments were carried out by using the already described microfluidic approach. The bare gold chip was modified by immersion in a DTSP solution ( $4 \mathrm{mM}$ in DMSO, 48 hours, under inert atmosphere and room temperature). DTSP is a homobifunctional, thiol-cleavable cross-linker that introduces a self-assembled monolayers of $\mathrm{N}$ succinimidyl-3-thiopropionate groups on the gold surface [43]. ASF surface immobilization was carried out by flowing ASF (1.04 $\mu \mathrm{M}$ in PBS) through parallel PDMS microchannels in contact to the DTSP modified gold surface. An oriented immobilization of the ASF with respect to $\beta$-linked galactosyl residue, resulting from its immobilization through the peptide moieties, reduced any steric hindrance between the surface and the Gal $\beta_{1,3}$ GalNAc residue [44]. Unreacted DTSP groups were deactivated by soaking the surface in an mPEG- $\mathrm{NH}_{2}$ solution ( $4 \mathrm{mM}$ in $0.1 \mathrm{M}$ TEA, $\mathrm{pH}=8$, for 2 hours). A set of four PDMS microchannels was used in order to study the interaction of $\mathrm{RCA}_{120}$ with the surface immobilized ASF. Four solutions having different $\mathrm{RCA}_{120}$ concentration (100 nM, $200 \mathrm{nM}, 400 \mathrm{nM}, 800 \mathrm{nM}$ in PBS $\mathrm{pH}=7.4$ ) were put in contact to the surface immobilized ASF (flow rate $50 \mu \mathrm{L} \mathrm{min}{ }^{-1}$ ). The experiments were replicated by introducing $\mathrm{ABs}$ between the running buffer and the $\mathrm{RCA}_{120}$ solutions. Kinetic parameters were obtained by fitting the SPRI experimental data according to procedures elsewhere described [45] on the basis of a 1:1 interaction model.

\section{RESULTS AND DISCUSSION}

In Fig. (1) the variation in percent reflectivity over time obtained for the same elution sequence with and without the introduction of an $\mathrm{AB}$ is reported. The difference in the refractive index of the two solutions $(\Delta \mathrm{n}=0.0006)$ [46] is registered by the SPRI apparatus with a change of about $\Delta \% \mathrm{R}$ $=2.8$. Although there is a clear-cut division between the two 
solutions at the entrance of the tubing system, in the absence of ABs this is not observed in the obtained SPRI response.

A time of about 100 seconds elapses before the switching between the two solutions is completed, i.e. diffusion occurred inside the tubing system and a sigmoidal lag phase (sample mixing) is observed in the SPRI response as a result of the creation of a gradient in the ethanol concentration within the liquid volume affected by the diffusion. Although the latter could be minimized by optimizing the experimental setup (length of the tubes, flow rate, use of switching valves at the entrance of the flow cell), it cannot be totally removed in a standard SPRI experiment. On the contrary, the introduction of $\mathrm{ABs}$ in the elution sequence removed the diffusion problem (Fig. (1), grey line). The size of the ABs could be controlled by varying the time of air aspiration by the peristaltic pump and 5 seconds was generally chosen as the best time interval for our experiments. Therefore, the $\mathrm{ABs}$ prevented the formation of a concentration gradient within the tubing system by maintaining the interface between two solutions constantly and completely separated.

For real biomolecular interactions, generally, whatever model is used for fitting the SPRI kinetic data, it is obvious that a gradient of the analyte concentration $(\Delta \mathrm{A})$ causes an SPRI response that can have negative effects on the interpretation of the data, altering the final value of the kinetics parameters obtained. $\Delta \mathrm{A}$ varies depending on the diffusion processes that occur in the tubing system, i.e. it depends on the diffusion coefficient of the analyte as well as on the particular conditions used for the experiment, so that the kinetics parameters calculated by fitting the detected SPRI response over time can be affected by errors. The latter will be higher for small molecules, as they diffuse rapidly into the buffer solutions. SPRI studies are often carried out on large biomolecules that, by having a low diffusion coefficient, create a small $\Delta \mathrm{A}$. Nevertheless, problems still arise if the interaction between the immobilized molecules and the flowing analyte is very fast. In this case, the initial slope of the detected SPRI response over time has an impact on the data fitting parameters, and therefore, in order to obtain reliable kinetics data, diffusion processes should be avoided. Although the $\mathrm{AB}$ method used in the case of the ethanol solution experiment previously described showed to be very successful for such purpose, the new experimental approach had to be verified in the case of a switching between a running buffer and a biomolecule containing solution. Furthermore, the potential damage that the contact with the air could cause to a SPRI sensor chip immobilized biomolecular system had to be investigated. A very strong interaction is established between the very well-known SA and biotin systems [47]. The value of $\mathrm{K}_{\mathrm{a}} \approx 10^{13} \mathrm{M}^{-1}$ is widely accepted for such a system as one of the highest affinity constant known in natural biological systems. It is then obvious that, in the study of the SA-biotin interaction, the first part of the SPRI response over time has a high impact on the kinetics parameters calculation and for this reason the SA-biotin interaction was chosen as a model system for our purposes. The SPRI kinetics data (Fig. 3) obtained from the interaction of surface-immobilized biotin with four solutions having different SA concentration were fitted by following the approach described in the Materials and Methods section. Although the SPRI signal is momentarily saturated during the ABs transit in the microchannel, this does not affect the overall result, as it is shown in
(Fig. 4). SPRI saturated signal returns to its original value after the AB transit, not affecting the SPRI kinetic data appearance during the initial stage of the interaction. Therefore, the SPRI data after the $\mathrm{AB}$ transit can be considered fully representative of the interaction and used for kinetics study.

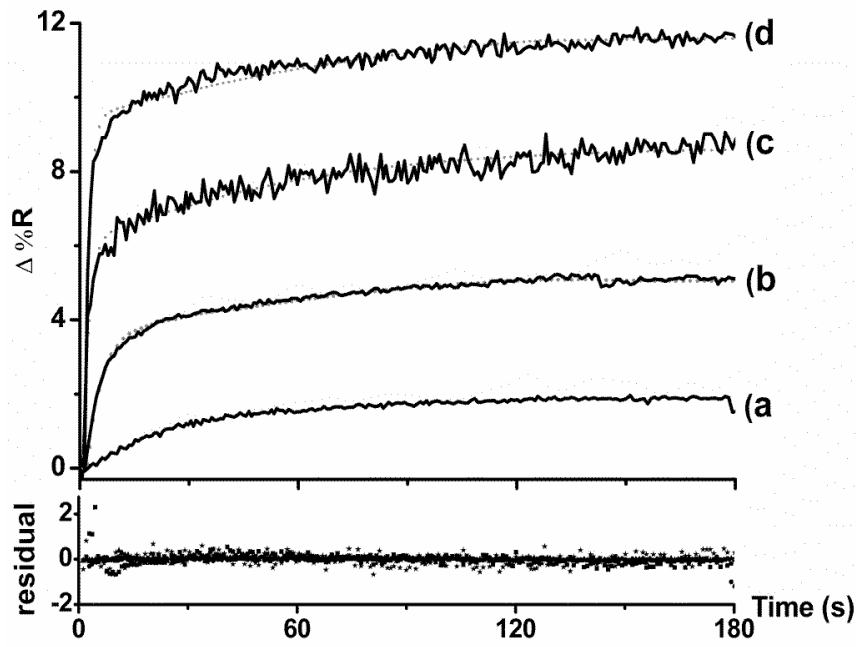

Fig. (3). Kinetics data for SA interaction with surface immobilized biotin. An $\mathrm{AB}$ was inserted between the running buffer and the SA solution. Four different concentrations of SA were used: $0.83 \mu \mathrm{M}$ (a), $1.66 \mu \mathrm{M}$ (b), $3.33 \mu \mathrm{M}$ (c), $5.83 \mu \mathrm{M}$ (d). Saturation of the signal due to the ABs transit in the microchannels was omitted for simplicity, while dashed lines represent fits obtained as described in the text. In the lower part of the graph the residuals are also reported.

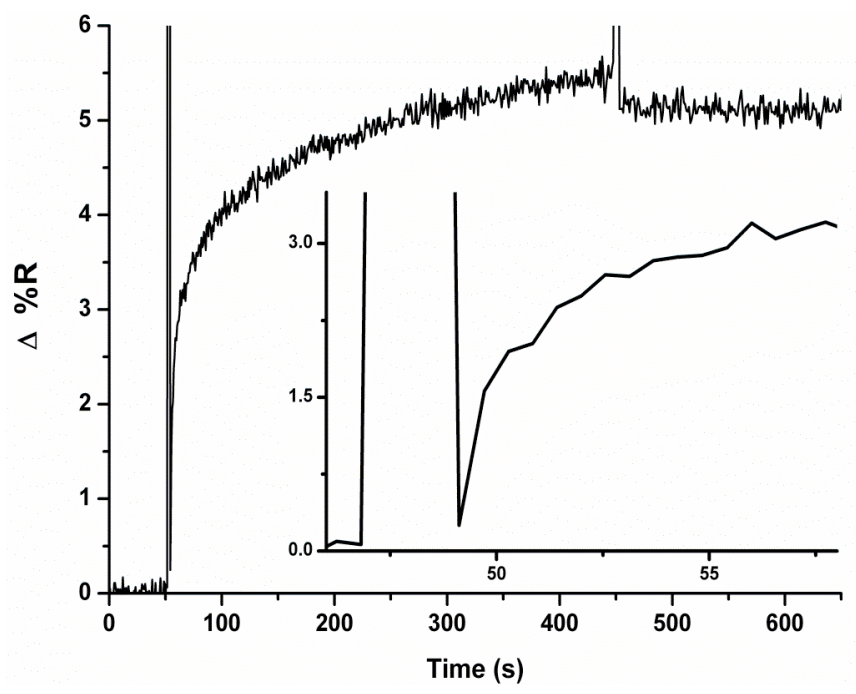

Fig. (4). Kinetics data for the elution sequence $[\mathrm{PBS} / \mathrm{SA}$ in $\mathrm{PBS} / \mathrm{PBS}]$. An AB was inserted between each change of solution, the surface was previously functionalized with biotin and a concentration of SA of $2.08 \mu \mathrm{M}$ was used. The zoomed area shows that the SPRI signal returns very close to its proper value after the saturation spike due to the ABs transit in the microchannel.

Analogous results are obtained if a comparison of SPRI data registered for SA solutions (SA concentration $2.08 \mu \mathrm{M}$ ) eluted with or without ABs (Fig. (5), gray line and black line respectively) is carried out. Also in this case, an SPRI response affected by an initial diffusion-produced sigmoidal lag-phase (Fig. (5), black line) is substituted with a diffu- 
sion-free exponential form when $\mathrm{ABs}$ are introduced in the elution sequence (Fig. (5), gray line).

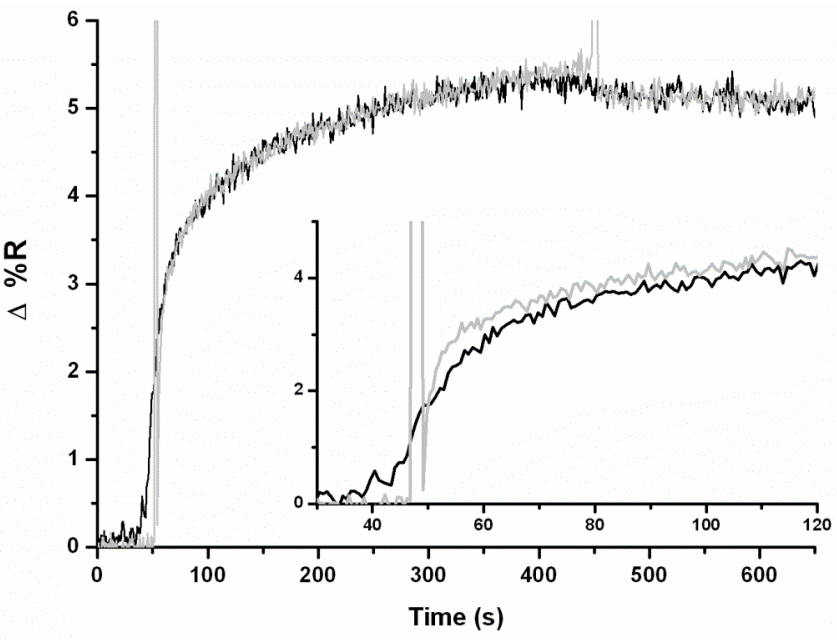

Fig. (5). Change in percent reflectivity over time for the elution sequence $[\mathrm{PBS} /(\mathrm{SA} 2.08 \mu \mathrm{M}$ in PBS)/PBS] with (grey line) and without (black line) the introduction of an AB. Differences in the SPRI short-time response (about 40 seconds for the shown experiment. See zoomed graph) cause changes that affect the evaluation of kinetic parameters. The introduction of an $\mathrm{AB}$ removes data artifacts due to concentration gradients inside the tubing system.

The average rate constants obtained by fitting the SPRI kinetic data for the adsorption of differently concentrated SA solutions (Fig. 3) are reported in Table $\mathbf{1}$ where results from different experimental approaches are compared. Before going into the details of the specific interaction, it is worth to highlight that the calculated kinetic parameters obtained from experiments where ABs were introduced in the elution sequence are similar to the ones reported in the literature, proving already the applicability of the new experimental protocol to the SA-biotin system. The AB transit did not cause a detectable alteration of the biotinylated surface as the latter would have produced a dramatic change of the kinetic parameters that was not observed. Kinetic parameters available from the literature $\left(\mathrm{k}_{\mathrm{on}}=5.13 \times 10^{6} \mathrm{M}^{-1} \mathrm{~s}^{-1}\right.$. See ref. [36] $)$ were calculated by assuming a simple bimolecular interaction model. Under analogous conditions data fit provided in our case a rather similar adsorption rate constant value $\left(\mathrm{k}_{\mathrm{on}}=\right.$ $\left.9.7 \times 10^{5} \mathrm{M}^{-1} \mathrm{~s}^{-1}\right)$. Data fitting carried out on the basis of the bivalent interaction model, resulting from the consideration that interaction can take place between the surface immobilized biotin molecules and one or two of the binding sites present on the side of the SA facing the surface, provided faster adsorption rate constants when the experiments were conducted by using ABs $\left(\mathrm{k}^{1}\right.$ on $=8.7( \pm 0.3) \times 10^{4} \mathrm{M}^{-1} \mathrm{~s}^{-1}, \mathrm{k}^{2}$ on $=2.9( \pm 0.4) \times 10^{3} \mathrm{M}^{-1} \mathrm{~s}^{-1}$. Table 1). As expected, the desorption rate constants $\left(\mathrm{k}^{1}\right.$ off and $\left.\mathrm{k}_{\text {off }}^{2}\right)$ obtained by considering only kinetic data for the SA adsorption are seriously affected by limitations imposed by the high affinity of the interaction and significantly differ from values reported in the literature from both solution $\left(\mathrm{k}_{\mathrm{off}}=5.4 \times 10^{-6} \mathrm{~s}^{-1}\right)$ [24] as well as conventional SPR desorption experiments $\left(\mathrm{k}_{\mathrm{off}}=1.4 \times 10^{-4} \mathrm{~s}^{-1}\right.$ [35]; $\left.\mathrm{k}_{\mathrm{off}}=1.4 \times 10^{-5} \mathrm{~s}^{-1}[34]\right)$.

Although difficulties in comparing SPR data due to the different procedures adopted to immobilize biotin molecules onto the gold surface and to the different values of surface coverage of biotin are expected, it is important to note that similar calculations carried out by considering SPRI data obtained from experiments conducted with no ABs produce results which testify the alteration of the faster adsorption rate constant $\left(\mathrm{k}_{\text {on }}^{1}=1.6( \pm 0.3) \times 10^{4} \mathrm{M}^{-1} \mathrm{~s}^{-1}\right)$ due to the liquid-liquid diffusion lag. The calculated value produces an underestimation of the value obtained from experiments conducted with ABs.

To better evaluate the contribution of the desorption onto the SA-biotin equilibrium and in order to further prove the conserved integrity of the biological system we decided to run experiments where the SA-biotin interaction was followed for few hours in the desorption phase. Due to the exceptionally high affinity of SA for biotin, SA was desorbed from surface-bound SA molecules by competitive dissociation with free biotin in solution. This was achieved by the injection of $1 \mathrm{mM}$ free biotin in PBS buffer at the end of injection of the SA sample and the dissociation of SA was monitored for few hours. A similar experimental condition was also used to avoid rebinding of SA during the long-time desorption. The $\Delta \% \mathrm{R}$ variation over time obtained is reported in Fig. (6).

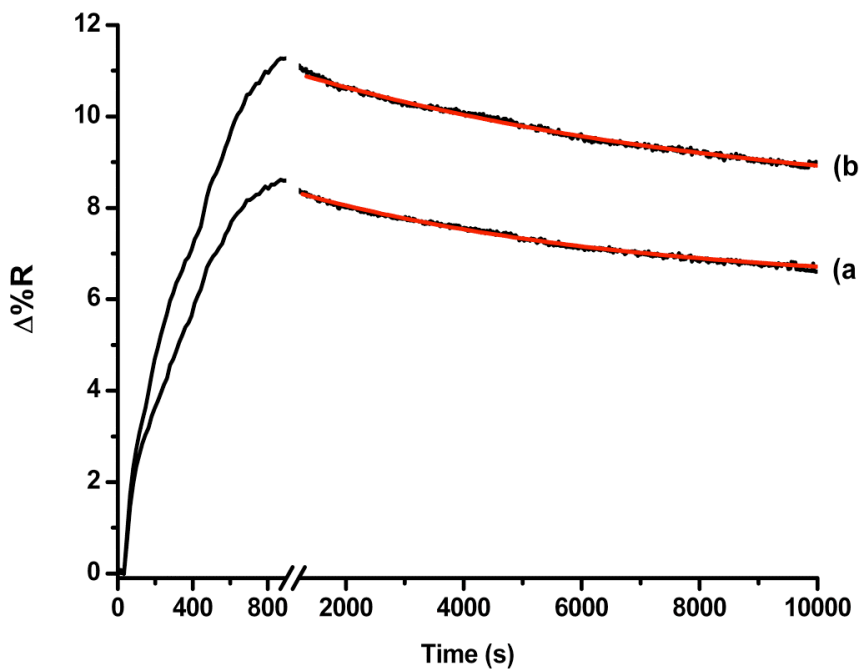

Fig. (6). Kinetics data for the desorption of surface bound SA by competitive dissociation with free biotin in solution ( $1 \mathrm{mM}$ biotin in PBS buffer). Streptavidin was immobilized at $3.33 \mu \mathrm{M}$ (a), $5.83 \mu \mathrm{M}$ (b) concentration on the biotinylated surface. Dissociation started at about $800 \mathrm{sec}$. Saturation of the signal due to the ABs transit in the microchannels was omitted for simplicity. The best fit $\left(\mathrm{R}^{2}=0.99\right)$ single first-order exponential decay desorption process (red line) led to a desorption rate constant value of $\mathrm{k}^{\text {app }}$ off $=1.5( \pm 0.4) \times 10^{-4} \mathrm{~s}^{-1}$.

According to procedures described elsewhere [35], SPRI kinetic data for the long-time desorption of SA from the biotinylated surface were fitted by using a single first-order exponential decay equation. An average value of dissociation rate constant of $\mathrm{k}^{\mathrm{app}}$ off $=1.5( \pm 0.4) \times 10^{-4} \mathrm{~s}^{-1}$ was obtained in this case. This value is in the range of values reported for the SA desorption process from biotinylated surfaces (Table 1) $[34,35]$.

These results were considered a confirmation that the described method can be safely applied to biological systems and that ABs inside the flow channels do not cause any SPRI detectable damage to the surface immobilized biotin (as confirmed by the results reported in Fig. (4)). Moreover, the 
calculated kinetic rate constant values are in the expected range for the SA-biotin interaction, demonstrating that no SPRI detectable damage to the biomolecules system occurred. Moreover, different fitting procedures and data evaluations helped to support the conclusion that SA interacts with biotin by establishing equilibria with rate constants that have values within the expected range for such a system.

In order to further prove the applicability of the $A B$ method to real biomolecular systems, the interaction of $\mathrm{RCA}_{120}$ with the surface immobilized ASF was used as a second model system for the SPRI real-time binding kinetics monitoring. The chosen interaction is not as fast as the SAbiotin one and the diffusivity of $\mathrm{RCA}_{120}$ is lower than that of SA $\left(D_{\mathrm{SA}}=9.710^{-7} \mathrm{~cm}^{2} \mathrm{~s}^{-1} ; D_{\mathrm{RCA}}=7.510^{-7} \mathrm{~cm}^{2} \mathrm{~s}^{-1} . \mathrm{T}=25^{\circ} \mathrm{C}\right.$. Values calculated with the ClampXP software). Both the above mentioned points are expected to minimize the influence of the lag-phase over the SPRI kinetics data. Fig. (7) shows the SPRI data obtained when the ASF-RCA 120 interaction was investigated by using a specific $\mathrm{RCA}_{120}$ solution $\left(\mathrm{RCA}_{120}\right.$ concentration $\left.400 \mathrm{nM}\right)$. The experiments were carried out with or without $\mathrm{AB}$ (gray line and black line respectively.). Also in this case the initial diffusion-produced sigmoidal lag-phase (Fig. (7), black line) is substituted with a diffusion-free exponential form (Fig. (7), gray line) but the advantage offered by the diffusion-free environment is

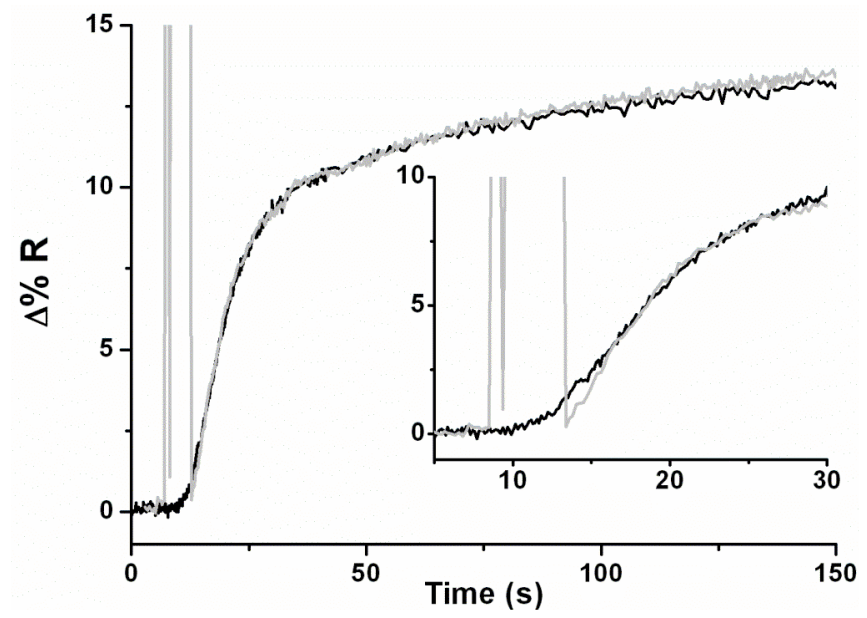

Fig. (7). Change in percent reflectivity over time for the elution sequence $\left[\mathrm{PBS} /\left(\mathrm{RCA}_{120} 400 \mathrm{nM}\right.\right.$ in $\left.\left.\mathrm{PBS}\right) / \mathrm{PBS}\right]$ with (gray line) and without (black line) the introduction of an AB. Differences in the SPRI short-time response (about 30 seconds for the shown experiment. See zoomed graph) cause changes that affect the evaluation of kinetic parameters. The introduction of an $\mathrm{AB}$ removes data artifacts due to concentration gradients inside the tubing system.

minimized and very similar kinetic behaviors are to be predicted from the SPRI data evaluation. Kinetic SPRI experiments were carried out in order to further prove the conserved integrity of the surface immobilized biological system after the AB transit. Fig. (8) shows SPRI kinetic data obtained from the interaction of surface-immobilized ASF with four solutions having different $\mathrm{RCA}_{120}$ concentration $(100 \mathrm{nM}, 200$ $\mathrm{nM}, 400 \mathrm{nM}, 800 \mathrm{nM}$ in PBS $\mathrm{pH}=7.4)$. The SPRI kinetic data for the interaction of ASF with a $400 \mathrm{nM} \mathrm{RCA}_{120}$ solution obtained without $\mathrm{AB}$ are shown for comparison (Fig. (8), red line). The $\Delta \% \mathrm{R}$ variation over time following the signal saturation due to the $\mathrm{AB}$ transit was used to calculate the rate con- stants (Table 2). The calculated values are in agreement with data reported in the literature [40] even if a slower dissociation rate was observed in our case $\left(\mathrm{k}_{\text {off }}=3.48( \pm 0.02) \times 10^{-4} \mathrm{~s}^{-1}\right.$ to be compared to $\left.\mathrm{k}_{\text {off }}=2.1( \pm 0.1) \times 10^{-3}[41]\right)$. The difference cannot be a consequence of an $\mathrm{AB}$ generated surface degradation since a quite similar SPRI kinetic response was observed when no ABs were used (Fig. 8). However, different experimental parameters have been reported to significantly affect the dissociation rate constants for the $\mathrm{RCA}_{120}$ interaction with human erythrocytes [48].

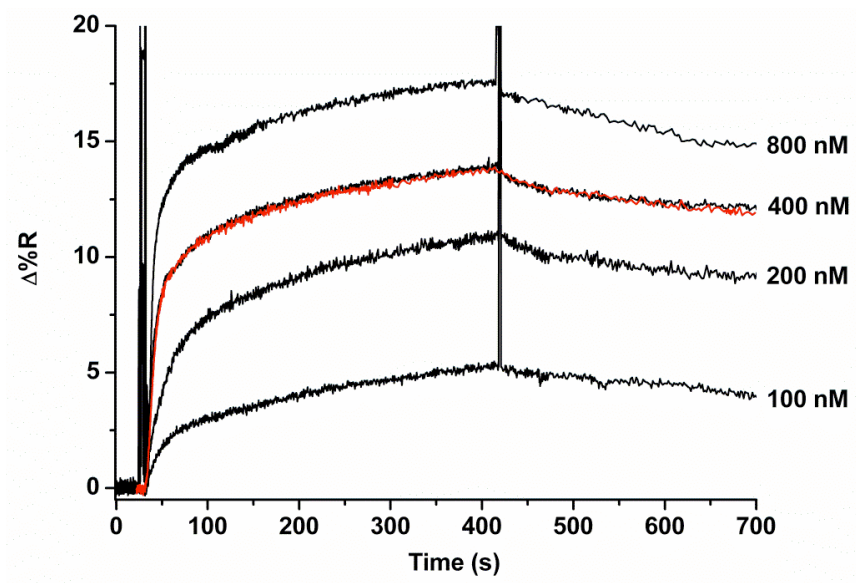

Fig. (8). SPRI kinetic data obtained from the interaction of surfaceimmobilized ASF with four solutions having different $\mathrm{RCA}_{120}$ concentration (100 nM, $200 \mathrm{nM}, 400 \mathrm{nM}, 800 \mathrm{nM})$. The SPRI kinetic data for the interaction of ASF with a $400 \mathrm{nM} \mathrm{RCA}_{120}$ solution obtained without $\mathrm{AB}$ are shown for comparison (red line).

The above reported discussion for both SA-biotin and ASF-RCA ${ }_{120}$ interactions shows that it can be quite difficult to compare SPRI results from different experimental procedures. Nevertheless, we believe that our results showed a significant advantage in using the new $\mathrm{AB}$ approach, which is clearly demonstrated by comparing kinetic constants obtained within the same SPRI setup for different biomolecular systems.

Table 1. Adsorption $\left(k_{0 n}\right)$ and Desorption $\left(k_{\text {off }}\right)$ Kinetic Rate Constants for SA-Biotin System Obtained by Different Experimental and Fitting Procedures, as Described in the Text

\begin{tabular}{|c|c|c|c|c|}
\hline & $\mathbf{k}^{1}{ }_{\text {on }}\left(M^{-1} \mathbf{s}^{-1}\right)$ & $k^{1}{ }_{\text {off }}\left(s^{-1}\right)$ & $k^{2}{ }_{o n}\left(M^{-1} s^{-1}\right)$ & $k_{\text {off }}^{2}\left(s^{-1}\right)$ \\
\hline $\begin{array}{l}\text { Adsorption } \\
\text { with } \mathrm{AB}\end{array}$ & $\begin{array}{c}(8.7 \pm 0.3) \\
\times 10^{4}\end{array}$ & $\begin{array}{c}(7.2 \pm 0.5) \\
\times 10^{-2}\end{array}$ & $(2.9 \pm 0.4) \times 10^{3}$ & $(6.9 \pm 0.3) \times 10^{-3}$ \\
\hline $\begin{array}{l}\text { Adsorption } \\
\text { without } A B\end{array}$ & $\begin{array}{c}(1.6 \pm 0.3) \\
\times 10^{4}\end{array}$ & $\begin{array}{c}(2.9 \pm 0.9) \\
\times 10^{-2}\end{array}$ & $(1.3 \pm 0.5) \times 10^{3}$ & $(3.3 \pm 0.2) \times 10^{-3}$ \\
\hline $\begin{array}{l}\text { Long- Time } \\
\text { Desorption }\end{array}$ & & $\begin{array}{c}(1.5 \pm 0.4) \\
\times 10^{-4 a}\end{array}$ & & \\
\hline Literature & $5.13 \times 10^{6 \mathrm{~b}}$ & $\begin{array}{l}5.4 \times 10^{-6 \mathrm{c}} \\
1.4 \times 10^{-4 \mathrm{~d}} \\
1.4 \times 10^{-5} \mathrm{e}\end{array}$ & & \\
\hline
\end{tabular}

$\mathrm{k}^{1}$ and $\mathrm{k}^{2}$ refer to kinetic constant calculated according to a bivalent interaction model that consider the SA interaction with one $\left(\mathrm{k}^{1}\right)$ or two $\left(\mathrm{k}^{2}\right)$ biotin molecules respectively. ${ }^{\mathrm{a}} \mathrm{k}^{\mathrm{app}}{ }_{\text {off }}$ obtained by fitting with a single first-order exponential decay equation. ${ }^{\mathrm{b}}$ From ref. [36]. Value obtained by using a 1:1 interaction model. $\mathrm{k}_{\text {off }}$ was not determined in this paper. ${ }^{\mathrm{c}}$ From ref. [24]. Value obtained from solutions. ${ }^{\mathrm{d}}$ From ref. [35]. Desorption rate constant reported as $\mathrm{k}^{\text {app }}{ }_{\text {off. }}{ }^{\mathrm{e}}$ From ref. [34]. 
Table 2. Adsorption ( $k_{\text {on }}$ ), Desorption ( $k_{\text {off }}$ ) Rate Constants and Affinity $\left(K_{A}\right)$ Constant for ASF-RCA ${ }_{120}$ System Obtained by Introducing $\mathrm{ABs}$ in the Elution Sequence as Explained in the Text

\begin{tabular}{|c|c|c|c|}
\hline & $\mathbf{k}_{\text {on }}\left(\mathbf{M}^{-1} \mathbf{s}^{-1}\right)$ & $\mathbf{k}_{\text {off }}\left(\mathbf{s}^{-1}\right)$ & $\mathbf{K}_{\mathbf{A}}\left(\mathbf{M}^{-1}\right)$ \\
\hline \hline $\begin{array}{c}\text { Adsorption } \\
\text { with AB }\end{array}$ & $(2.27 \pm 0.03) \times 10^{5}$ & $(3.48 \pm 0.02) \times 10^{-4}$ & $(6.5 \pm 0.09) \times 10^{8}$ \\
\hline Literature $^{\mathrm{a}}$ & $(3.4 \pm 0.1) \times 10^{5}$ & $(2.1 \pm 0.1) \times 10^{-3}$ & $(1.6 \pm 0.1) \times 10^{8}$ \\
\hline
\end{tabular}

\section{CONCLUSIONS}

We have shown that the diffusion inside the tubing system leads to misleading SPRI data processing. When fast interaction processes and/or analytes with high diffusion coefficient are going to be investigated by SPRI, problems of altered kinetics parameters arise and the first part of the SPRI kinetic response is the most affected by the concentration gradient formed inside the tubing system. The introduction of $\mathrm{ABs}$ in the elution sequence completely removes the formation of a concentration gradient and liquid-liquid diffusion-free responses are produced. Different elution sequences were tested in order to assess the applicability of the new experimental procedure. For the elution sequence where water was switched to a water solution containing ethanol $(1 \%)$ the diffusion between the two solutions was completely removed and a perfect clear-cut step was observed in the SPRI response curve. Analogous results were obtained when a switching between a running buffer and a protein buffered solution was carried out.

The new experimental method was applied to the SAbiotin and the ASF-RCA 120 systems and the relevant SPRI kinetic data were used in order to calculate the kinetics parameters characteristic for the specific biomolecular interactions. The acceptable accordance with the data reported in the literature demonstrated that the biomolecules are not disrupted by $\mathrm{ABs}$, showing that the new approach can be applied to real biological systems.

This work demonstrated that in order to produce SPRI kinetic parameters that approach diffusion-free data, the use of the ABs procedure coupled with a proper experimental setup (high flow rate, use of microchannels, etc.) is revealed to be advantageous.

\section{ACKNOWLEDEGMENT}

We thank Dr. S. Weibel from GWC Technologies for comments and helpful discussions. This work was supported by MIUR (PRIN 2005 n 2005038704_004).

\section{REFERENCES}

[1] Terry SC, Jerman JH, Angell JB. Thin-Film Semiconductor NO Sensor. IEEE Trans Electron Devices ED 1979; 26: 1880-6.

[2] Whitesides GM. The origins and the future of microfluidics. Nature 2006; 442: 368-73.

[3] Squires TM, Quake SR. Microfluidics: Fluid physics at the nanoliter scale. Rev Mod Phys 2005; 77: 977-1026.

[4] El-Ali J, Sorger PK, Jensen KF. Cells on chips. Nature 2006; 442: 403-11.

[5] Yang J, Huang Y, Wang XB, Becker FF, Gascoyne PR. Cell separation on microfabricated electrodes using dielectropho- retic/gravitational field-flow fractionation. Anal Chem 1999; 71: 911-18.

[6] Simpson PC, Roach D, Woolley AT, et al. High-throughput genetic analysis using microfabricated 96-sample capillary array electrophoresis microplates. Proc Natl Acad Sci USA 1998; 95: 2256-61. Bökenkam D, Desai A, Yang X, Tai Y, Marzluff E, Mayo S. Microfabricated silicon mixers for submillisecond quench-flow analysis. Anal Chem 1998; 70: 232-26.

Hadd AG, Jacobson SC, Ramsey JM. Microfluidic Assays of Acetylcholinesterase Inhibitors. Anal Chem 1999; 71: 5206-12.

De Mello AJ. Control and detection of chemical reactions in microfluidic systems. Nature 2006; 442: 394-402.

Wegner GJ, Lee HJ, Corn RM. 2004. Surface plasmon resonance imaging measurements of DNA, RNA, and protein interactionts to biomolecular arrays. In: Kambhampati D, Ed. Protein Microarray Technology, John Wiley \& Sons, Inc., New York, 2003; pp. 10729.

[11] Shumaker-Parry JS, Campbell CT. Quantitative methods for spatially resolved adsorption/desorption measurements in real time by surface plasmon resonance microscopy. Anal Chem 2004; 76: 907 17.

[12] Rothenhäusler B, Knoll W. Surface-plasmon microscopy. Nature 1988; 332: 615-7.

[13] Wegner GJ, Wark AW, Lee HJ, et al. Real-time surface plasmon resonance imaging measurements for the multiplexed determination of protein adsorption/desorption kinetics and surface enzymatic reactions on peptide microarrays. Anal Chem 2004; 76: 5667-74.

[14] D'Agata R, Grasso G, Iacono G, Spoto G, Vecchio G. Lectin recognition of a new SOD mimic bioconjugate studied with surface plasmon resonance imaging. Org Biomol Chem 2006; 4: 610-12.

[15] Schuck P, Minton AP. Analysis of mass transport-limited binding kinetics in evanescent wave biosensors. Anal Biochem 1996; 240: 262-72.

[16] Christensen LLH. Theoretical analysis of protein concentration determination using biosensor technology under conditions of partial mass transport limitation. Anal Biochem 1997; 249: 153-64.

[17] Myszka DG, He X, Dembo M, Morton TA, Goldstein B. Extending the range of rate constants available from BIACORE: interpreting mass transport-influenced binding data. Biophys J 1998; 75: 58394.

[18] Abrantes M, Magone MT, Boyd LF, Schuck P. Adaptation of a Surface Plasmon Resonance Biosensor with microfluidics for use with small sample volumes and long contact times. Anal Chem 2001; 73: 2828-35.

[19] Jennisen HP, Zumbrink T. A novel nanolayer biosensor principle. Biosens Bioelect 2004; 19: 987-97.

[20] Navratilova I, Eisenstien E, Myszka DG. Measuring long association phases using Biacore. Anal Biochem 2005; 344: 295-7.

[21] Sonksen CP, Roepstorff P, Markgren P, Danielson UH, Hamalainen MD, Jansson O. Capture and analysis of low molecular weight ligands by surface plasmon resonance combined with mass spectrometry. Eur J Mass Spectrom 2001; 7: 385-91.

[22] Nelson BP, Frutos AG, Brockman JM, Corn RM. Near-Infrared surface plasmon resonance measurements of ultrathin films. 1. Angle shift and SPR imaging experiments. Anal Chem 1999; 71: 3928-34.

[23] Grasso G, Fragai M, Rizzarelli E, Spoto G, Yeo KJ. In Situ APMALDI characterization of anchored MMPs. J Mass Spectrom 2006; 41: 1561-69.

[24] Chilkoti A, Stayton PS. Molecular origins of the slow StreptavidinBiotin dissociation kinetics. J Am Chem Soc 1995; 117: 10622-28.

[25] Wilchek M, Bayer EA. The avidin-biotin complex in bioanalytical applications. Anal Biochem 1988; 171: 1-32.

[26] Wilchek M, Bayer EA. Introduction to avidin-biotin technologyApplications of avidin-biotin technology: literature survey. Methods Enzymol 1990; 184: 5-13, 14-45.

[27] Hall DR, Cann JR, Winzor DJ. Demonstration of an upper limit to the range of association rate constants amenable to study by biosensor technology based on Surface Plasmon Resonance. Anal Biochem 1996; 235: 175-84.

[28] Yam CM, Pradier CM, Salmain M, Marcus P, Jaoueny G. Binding of biotin to gold surfaces functionalized by self-assembled monolayers of cystamine and cysteamine: combined FT-IRRAS and XPS characterization. J Colloid Interface Sci 2001; 235: 183-9. 
[29] Lee HJ, Goodrich TT, Corn RM. SPR imaging measurements of 1$\mathrm{D}$ and 2-D DNA microarrays created from microfluidic channels on gold thin films. Anal Chem 2001; 73: 5525-31.

[30] Ober RJ, Ward ES. The choice of reference cell in the analysis of kinetic data using BIAcore. Anal Biochem 1999; 271: 70-80.

[31] Sano T, Cantor CR. Cooperative biotin binding by streptavidin. Electrophoretic behavior and subunit association of streptavidin in the presence of $6 \mathrm{M}$ urea. J Biol Chem 1990; 265: 3369-73.

[32] González M, Bagatolli LA, Echabe I, et al. Interaction of Biotin with Streptavidin. Thermostability and conformational changes upon binding. J Biol Chem 1997; 272: 11288-94.

[33] Hendrickson WA, Pahler A, Smith JL, Satow Y, Merritt EA, Phizackerley RP. Crystal structure of core streptavidin determined from multiwavelength anomalous diffraction of synchrotron radiation. Proc Natl Acad Sci USA 1989; 86: 2190-4.

[34] Jung LS, Nelson KE, Stayton PS, Campbell CT. Surface plasmon resonance measurement of binding and dissociation of wild-type and mutant streptavidin on mixed biotin-containing alkylthiolate monolayers. Langmuir 2000; 16: 9421-32.

[35] Pérez-Luna VH, O’Brien MJ, Opperman KA, et al. Molecular recognition between genetically engineered streptavidin and surface-bound biotin. J Am Chem Soc 1999; 121: 6469-78.

[36] Qureshi MH, Yeung JC, Wu SC, Wong SL. Development and Characterization of a Series of Soluble Tetrameric and Monomeric Streptavidin Muteins with Differential Biotin Binding Affinities. J Biol Chem 2001; 276: 46422-8.

[37] Tang Y, Mernaugh R, Zeng X. Nonregeneration protocol for surface plasmon resonance: study of high-affinity interaction with high-density biosensors. Anal Chem 2006; 78: 1841-8.

[38] Myszka DG, Morton TA. CLAMP ${ }^{\circ}$ a biosensor kinetic data analysis program. Trends Biochem Sci 1998; 23: 149-50.
[39] Ambrosi M, Cameron NR, Davis BG. Lectins: tools for the molecular understanding of the glycocode. Org Biomol Chem 2005; 3 : 1593-608.

[40] Cambi A, Figdor CG. Levels of complexity in pathogen recognition by C-type lectins. Curr Opin Immunol 2005; 17: 345-51.

[41] Shinohara Y, Kim F, Shimizu M, Goto M, Tosu M, Hasegawa Y. Kinetic measurement of the interaction between an oligosaccharide and lectins by a biosensor based on surface plasmon resonance. Eur J Biochem 1994; 223: 189-94.

[42] Spiro RG, Bhoyroo VD. Structure of the O-Glycosidically linked carbohydrate units of fetuin. J Biol Chem 1974; 249: 5704-17.

[43] Dordi B, Schönherr H, Vancso GJ. Reactivity in the confinement of self-assembled monolayers: chain length effects on the hydrolysis of N-Hydroxysuccinimide ester disulfides on gold. Langmuir 2003; 19: $5780-6$.

[44] Milton JD, Fernig DG, Rhodes JM. Use of a biosensor to determine the binding kinetics of five lectins for Galactosyl-Nacetylgalactosamine. Glycoconjug J 2001; 18: 565-9.

[45] Critchley P, Willand MN, Rullay AK, Crout DHG. Carbohydrateprotein interactions at interfaces: synthesis of thiolactosyl glycolipids and design of a working model for surface plasmon resonance. Org Biomol Chem 2003; 1: 928-38.

[46] Lide DR, Ed. CRC Handbook of Chemistry and Physics 87th Ed., Taylor and Francis, Boca Raton, 2006.

[47] Wilchek M, Bayer EA, Livnah O. Essentials of biorecognition: the (strept)avidin-biotin system as a model for protein-protein and protein-ligand interaction. Immun Lett 2006; 103: 27-32.

[48] Sandvig K, Olsnes S, Pihl A. Kinetics of binding of the toxic lectins abrin and ricin to surface receptors of human cells. J Biol Chem 1976; 251: 3977-84.

(c) D'Agata et al.; Licensee Bentham Open.

This is an open access article distributed under the terms of the Creative Commons Attribution License (http://creativecommons.org/license/by/2.5/), which permits unrestrictive use, distribution, and reproduction in any medium, provided the original work is properly cited. 